\title{
La régulation sociale par la violence : le rôle de la criminalité organisée dans l'Italie méridionale
}

\section{Raimondo Catanzaro}

\section{(2) OpenEdition \\ Journals}

Édition électronique

URL : http://journals.openedition.org/conflits/306

DOI : $10.4000 /$ conflits.306

ISSN : $1777-5345$

Éditeur :

CCLS - Centre d'études sur les conflits lilberté et sécurité, L'Harmattan

Édition imprimée

Date de publication : 15 mai 1993

ISSN : 1157-996X

\section{Référence électronique}

Raimondo Catanzaro, «La régulation sociale par la violence : le rôle de la criminalité organisée dans I'Italie méridionale », Cultures \& Conflits [En ligne], 09-10 | printemps-été 1993, mis en ligne le 13 mars 2006, consulté le 30 mars 2021. URL : http://journals.openedition.org/conflits/306 ; DOI : https:// doi.org/10.4000/conflits.306

Ce document a été généré automatiquement le 30 mars 2021

Creative Commons License 


\title{
La régulation sociale par la violence : le rôle de la criminalité organisée dans l'Italie méridionale
}

\author{
Raimondo Catanzaro
}

L'augmentation des activités illicites a atteint sa cote d'alerte dans la dernière décennie et la situation s'aggrave encore aujourd'hui. Si l'on regarde les données des statistiques criminelles relatives aux délits dénoncés, pour lesquels une action pénale a été engagée, nous pouvons vérifier un développement des homicides, en particulier dans les trois ou quatre régions à haute densité de criminalité organisée (Sicile, Calabre, Campanie et, depuis quelques années, également les Pouilles) et aussi une augmentation des délits contre le patrimoine, contre l'économie, la confiance publique et contre l'Etat. Les rapports d'ouverture de l'année judiciaire présentent chaque année un tableau plus sombre et l'on doit noter qu'ils se basent sur des données officielles qui présentent en ce domaine la faiblesse bien connue des "chiffres noirs", c'est-à-dire de ces délits qui échappent aux relevés statistiques, soit parce qu'ils ne sont pas dénoncés, soit parce qu'ils relèvent de l'administration d'une justice, autre que la justice officielle, à l'intérieur des groupes criminels ou des espaces qu'ils contrôlent. En outre, les récents assassinats d'hommes politiques d'importance à Palerme (comme le député au Parlement européen Lima) et de magistrats comme Falcone et Borsellino, qui avaient engagé de grandes batailles contre la mafia, ont fait croître les préoccupations quant aux dommages que la présence des groupes criminels peut causer en Italie et en Europe. Les questions auxquelles on voudrait répondre ici peuvent être formulées de la manière suivante: la croissance des comportements illégaux implique-t-elle une croissance de la "régulation par la violence" dans la société ? Si oui, pourquoi et à quelles conditions? Quels sont les modalités de comportements illégaux qui peuvent contribuer à l'augmentation de la régulation par la violence? Quelles variables interviennent pour favoriser ou contrarier cette forme de régulation sociale? Pour répondre à ces questions, il serait bon d'abandonner le thème excessivement large de l'illégalité diffuse et de se concentrer sur les groupes criminels. En d'autres termes, 
nous partirons d'une analyse de la "criminalité organisée" pour répondre au comment et au pourquoi de la croissance de la régulation sociale par la violence.

\section{DEUX FACES DE LA CRIMINALITE}

Dans le débat qui se déroule depuis longtemps déjà en Italie sur la mafia, notamment à partir des études américaines sur la criminalité organisée (organized crime), sont confondues les deux principales activités des groupes criminels, qu'il convient de garder analytiquement distinctes, tant pour en comprendre le fonctionnement que pour en évaluer de manière adéquate la dangerosité. Pour saisir la nature et les caractéristiques des deux activités, nous pouvons prendre l'exemple du trafic de drogue (mais cela vaut pour tout type de trafic illicite). Un groupe criminel qui doit faire décharger une quantité d'héroïne ou de cocaïne dans un port ou un aéroport quelconque, doit obtenir l'assentiment du groupe qui contrôle les activités illicites du lieu. La découverte du trafic pourrait en effet créer des problèmes au groupe qui contrôle le port, lequel pourrait se venger des trafiquants en les dénonçant à la police, en empêchant des trafics ultérieurs ou, cas extrême, en en tuant quelques uns. Au contraire, l'approbation du groupe criminel qui contrôle le port de transit des marchandises, garantit contre de tels risques. En outre, le groupe de contrôle sait quels sont les lieux et les modes les plus sûrs pour faire transiter les marchandises; il est en mesure de compter sur la bienveillance des policiers qui ne sont pas mal disposés ou sont même corrompus. Il est donc avantageux pour les trafiquants d'informer le groupe qui contrôle ce territoire et d'obtenir son accord qui, d'ailleurs, est payé en pourcentage du chiffre d'affaires. Cette quote-part peut être interprétée, pour le groupe des trafiquants, comme une forme d'assurance contre un double risque : celui d'être découverts par la police ou par le groupe qui contrôle le territoire. Pour ce dernier, le paiement peut être interprété de deux façons: d'un côté comme une compensation pour la prestation de services et pour les coûts engagés (qui incluent ceux relatifs à la couverture des forces de l'ordre); de l'autre comme une forme de monétarisation du risque lié au fait qu'une augmentation du volume des trafics illicites pourrait dépasser le seuil retenu comme acceptable par la police et la conduire à intervenir, avec les complications consécutives pour l'ensemble des affaires illicites. Réfléchissons aux caractéristiques des deux groupes: le premier, celui qui organise le trafic de drogue, peut être défini comme un entrepreneur économique qui produit et/ ou distribue une marchandise, bien qu'il s'agisse d'une marchandise dont la production et la distribution est interdite par la loi. Le second a une nature complètement différente : c'est un entrepreneur politique qui contrôle un territoire et exige un tribut pour toutes les affaires qui s'y déroulent. Nous devons distinguer de façon analytique entre activités économiques illicites (trafic de drogue, contrebande de tabacs, organisation et exploitation de la prostitution, jeux de hasard, loto clandestin) d'un côté, et contrôle politico-militaire d'un territoire de l'autre. La distinction a peut-être été formulée de la manière la plus efficace par A. Block[East Side, West Side. Organizing Crime in New York 1930-1950, Cardiff, University College Press, 1980.] avec les concepts d'enterprise syndicate et power syndicate, et c'est une distinction d'un grand intérêt car les activités qui se déroulent dans les deux camps répondent à des logiques tout à fait opposées. Les activités économiques illicites sont réalisées avec des critères de 
marché, bien qu'il s'agisse d'un marché très particulier: celui de la production et distribution de biens qui doivent circuler de manière occulte pour échapper à la sanction de la loi. Cela a conduit certains auteurs [P. Reuter, Disorganized Crime. The Economics of the Visible Hand, Cambridge (Mass.), MIT Press, 1983.] à opposer de façon polémique, en ce domaine, le concept de "criminalité désorganisée" à celui, courant, de criminalité organisée. Même si le concept de criminalité désorganisée, comme simple anarchie des comportements de marché, semble surtout répondre à une configuration idéal-typique, il ne fait pas de doute cependant que, dans les secteurs considérés, même s'il y a des formes d'alliances et de cartel pour le contrôle du marché, la règle fondamentale des comportements des acteurs est la concurrence. En ce qui concerne le power syndicate, dont les formes les plus apparentes dans le cas de la criminalité organisée du Mezzogiorno sont les extorsions et les séquestrations de personnes, nous nous trouvons en revanche devant un exemple classique de spécialisation politique. Le but fondamental du power syndicate est le contrôle de l'environnement et des individus qui en font partie; et ainsi, de ce point de vue, les mafiosi ont été définis comme des power brokers[A. Blok, The Mafia of a Sicilian Village, 1860-1960, New York, Harper, 1974.].

\section{LA PROTECTION-EXTORSION}

Une des activités fondamentales des power brokers criminels consiste dans l'extorsion d'argent en échange de protection. L'exemple cité auparavant à propos du groupe criminel qui contrôle une aire territoriale entière, est un cas d'implantation dont la genèse est constituée par l'extorsion. Analysons donc le mécanisme de l'extorsion pour reconstituer le système du contrôle sur les individus et le milieu. A la différence des délits comme le vol ou l'attaque à main armée, l'extorsion est un délit continu (cette caractéristique est également présente dans la séquestration à fin d'extorsion, mais elle se termine avec la libération de la victime). Nous ne nous intéressons pas ici aux dimensions juridiques de la continuité, mais à ses dimensions sociales. Comme l'extorsion ne s'épuise pas dans un acte unique et demande un rapport constant avec la victime, cette dernière doit être continuellement manipulée, ainsi que ses réseaux de sociabilité. De la même manière, doivent être manipulés la loi et ses représentants. En fait le mafioso doit, en même temps, menacer et protéger sa victime : il doit donc être en mesure d'exercer un pouvoir effectif envers ceux qui prétendent intervenir dans ce rapport: les autres criminels ou l'autorité publique. En d'autres termes, il doit savoir exploiter au maximum ses positions stratégiques dans une série de réseaux sociaux. Mais, en définitive, le fondement de sa capacité de protection et donc d'extorsion, réside dans le potentiel de violence que l'auteur de la protection/extorsion est en mesure d'exercer. Nous pouvons donc définir les acteurs de la criminalité organisée, envisagés comme power broker ou power syndicate, comme des sujets qui se spécialisent dans l'offre de protection privée et dont le financement se fait sur la base de prestations extorquées qui prennent un caractère de versements stables et périodiques en échange de l'affectation de prestations parmi lesquelles la fourniture d'une garantie de sécurité est fondamentale[Max Weber, Economia e società, Milano, Ed. Comunità, 1964, Vol. 1, p 195.]. Il s'agit par conséquent de sujets qui concurrencent l'Etat ou offrent un bien que l'Etat n'est pas en mesure d'offrir. Quelles sont les 
conditions qui doivent être satisfaites pour offrir une protection? S'ils veulent se substituer à l'autorité de l'Etat comme garant des biens et de la sécurité physique des personnes, ceux qui se spécialisent dans ces activités doivent démontrer qu'ils savent exercer la force de manière plus efficace que les concurrents potentiels. Ils doivent donc se construire leur propre appareil de coercition physique et avoir le monopole de la violence sur un territoire déterminé[Cf. sur ce point Cultures \& Conflits, l'Harmattan, $\mathrm{n}^{\circ} 3$, automne 1991.]. Toutefois, si le titulaire de la violence obtenait effectivement la position de monopole, toute demande de protection adressée à des sujets privés viendrait à tomber et, partant, les mafiosi ne pourraient plus exiger des pots-de-vin ou des récompenses ad hoc pour la dispenser. En fait, une personne privée qui obtiendrait le monopole de la violence sur un territoire déterminé finirait par devenir un agent étatique. En d'autres termes, celui qui a le monopole de la violence doit offrir la protection comme un bien public, c'est-à-dire sans paiement de contre-prestations directes. Au contraire, à la différence de l'Etat qui offre la protection comme un bien public à tous les citoyens, la criminalité organisée est un entrepreneur en matière de protection. Un des buts des entrepreneurs est de maintenir élevée la demande de la marchandise offerte. A l'instar des entreprises qui font la promotion de leurs produits, les mafiosi recourent également au message publicitaire; ils doivent faire en sorte que la violence soit toujours présente sur leur marché, c'est-à-dire que la demande de protection ne faiblisse jamais. A cette fin, ils utilisent leur propre capacité à exercer la violence ; ils menacent les individus dans leur sécurité physique ou leur patrimoine et, en même temps, ils offrent leur protection contre cette menace en échange de pots-devin, tributs et autres formes de contribution économique. Les entrepreneurs de protection privée doivent par conséquent être en même temps entrepreneurs de violence; pour que leur offre de protection puisse être efficace et avoir un attrait, il doit toujours exister une demande de protection privée. C'est-à-dire qu'il doit exister un sujet : l'Etat qui, sur le papier, a le monopole de la violence légitime mais qui n'est pas en mesure de l'exercer efficacement, c'est-à-dire de protéger les citoyens. Ces considérations servent d'ailleurs à expliquer pourquoi la criminalité organisée n'est pas un anti-Etat et peut prospérer seulement lorsqu'il y a un Etat, mais un Etat inefficace. En fait, c'est seulement lorsqu'il existe un mécanisme de protection publique qui ne fonctionne pas que les conditions propices à l'apparition d'une protection privée peuvent se créer[Sur le processus historique de formation de la mafia dans la genèse de l'Etat national italien, voir R. Catanzaro Men of Respect. A Social History of the Sicilian Mafia, New York, Free Press, 1992.]. En revanche, si la protection publique n'existait même pas sur le papier, s'agenceraient des mécanismes qui, à la longue, induiraient une sélection entre les différents acteurs privés qui se concurrencent dans l'offre de protection jusqu'à ce que l'un d'entre eux émerge pour s'affirmer comme celui qui offre une protection comme un bien public. En fait, ceci a été historiquement le processus de formation de l'autorité publique dans les Etats nationaux.

\section{LA REGULATION SOCIALE PAR LA VIOLENCE}

Mais retournons à notre sujet. Nous avons défini les groupes de criminalité organisée, spécialisés dans la dimension power syndicate, comme des professionnels de la protection-extorsion. En d'autres termes, ils sont spécialistes de la manipulation des 
personnes et groupes sociaux ainsi que de la violence. Puisqu'ils assurent le contrôle du territoire, il s'agit de groupes et d'acteurs qui se spécialisent dans la régulation sociale violente. Reprenons maintenant l'exemple retenu initialement sur les rapports entre les deux types de groupes criminels. De cet exemple, nous pouvons déduire que les sujets du power syndicate n'exigent pas seulement des tributs des honnêtes citoyens, mais également des autres criminels. De la même manière que la croissance des activités économiques sur les marchés légaux demande des formes de réglementation toujours plus complexes, la croissance des activités économiques illicites fait augmenter la demande de gouvernement de la criminalité. Une telle demande est à la base de la croissance des power syndicates c'est-à-dire des groupes qui se spécialisent dans la régulation sociale par la violence. L'augmentation de la sphère des activités illicites comporte donc une augmentation de la sphère d'influence et une multiplication des power syndicates ; cela implique en conséquence une croissance des formes, de l'extension et de la quantité de la régulation sociale basée sur la violence. Pour comprendre cette croissance, il est nécessaire d'approfondir l'analyse des fonctions liées à l'aspect power syndicate des mafiosi. Fondamentalement, ces fonctions sont doubles : fonction d'entrepreneurs de protection violente et fonction de médiateurs sociaux ou power brokers. Les activités de médiation peuvent être remplies en occupant simplement des positions déterminées, c'est-à-dire en se plaçant sur les connexions cruciales des réseaux sociaux. La compétition entre les aspirants médiateurs consiste donc à conquérir les articulations les plus importantes dans les réseaux de relations interpersonnelles. Cela se produit avec la compétition pour instaurer des contacts personnels, des échanges, des rapports d'amitié et d'affaires. Mais quand, dans le monde de la criminalité, on en arrive à la reddition des comptes, le moyen le plus efficace pour triompher dans la compétition consiste dans l'élimination physique de l'adversaire. Les fréquents homicides provoquent une instabilité substantielle et continue des rapports sociaux. Une telle instabilité fait que la confiance ne peut jamais être tenue pour acquise de la part de quiconque : victimes de l'extorsion, spécialistes de la protection ou simples spectateurs ; la violence est donc perçue comme une option toujours possible et, en définitive, normale. La présence quotidienne de la violence interdit l'affirmation de rapports de confiance de type impersonnel et affectivement neutres. Là où domine la violence les liens doivent par nécessité être forts, intenses et connotés dans un sens affectif. Il a, en fait, été souligné combien, dans le Mezzogiorno d'Italie et en particulier en Sicile, il existe une connotation instrumentale de l'amitié[Voir J. Schneider, P. Schneider, Culture and Political Economy in Western Sicily, New York, Academic Press, 1976. Sur la distinction entre amitié instrumentale et amitié expressive, voir A. Blok Kinship, Friendship and PatronClients Relations in Complex Societies, in M. Banton (Dir.) The Social Anthropology of Complex Societies, London, Tavistock, 1966.]. Définir "ami" tout sujet auquel on est socialement autorisé à demander une faveur, que ce soit l'obtention d'une prestation à laquelle on a droit ou la violation d'une loi de l'Etat, dénote l'exigence de conférer un caractère de confiance personnalisée aux relations sociales, parce que l'on ne peut pas se fier "spontanément", en s'en remettant au seul fonctionnement de normes impersonnelles. Peut-on dire que ce type de confiance connote des liens forts et non pas des liens faibles[Pour la distinction entre les deux types de liens, voir M.S. Granovetter, "The Strenght of Weak Ties", in American Journal of Sociology, 78, n 6, 1973, p 1360-1380.] ? Oui certainement, mais comme tous les liens forts connotés affectivement, il s'agit d'un lien fragile. La confiance en général n'est pas donnée une 
fois pour toutes et peut également se transformer en manque de confiance. En fait, les liens affectifs tendent à être exclusifs; donc la confiance qui en découle tend à se concentrer au sein de groupes restreints et n'est pas diffusée socialement. Dans le cas des activités illicites de la criminalité organisée, ce caractère restreint des réseaux de confiance est accentué par la nécessité d'éviter que les trafics ne soient découverts. Dès lors, la confiance connotée en termes affectifs et non impersonnels a comme caractéristique que le manque de respect à l'égard des engagements pris tend à revêtir l'aspect de la "trahison". Ceci sert à expliquer pourquoi dans le monde de la criminalité organisée le respect des engagements pris est aussi fortement connoté en termes d'"honneur", et combien la violation des engagements en vient à être considérée comme une insulte sur le plan personnel. Dans un contexte de relations interpersonnelles de ce type, toute altération des réseaux sociaux tendra à revêtir des connotations dramatiques très personnalisées. Non seulement la confiance se transformera brusquement en manque de confiance, mais la violence sera considérée comme une option et une riposte adéquate à la "trahison", c'est-à-dire à la violation des engagements. En conclusion de ce raisonnement, on peut affirmer que la croissance de la régulation sociale par la violence est fonction de la croissance des trafics illicites. Mais quels facteurs pèsent sur cette croissance ? Et existe-t-il une relation directe entre croissance des trafics illicites et croissance de la régulation sociale par la violence ? Ou devons-nous retenir qu'il existe des variables qui interviennent pour ne pas rendre linéaire ce rapport? Pour donner une réponse à ces demandes, nous prendrons deux points en considération. Le premier se réfère aux conséquences des activités illicites sur le marché et les comportements des entrepreneurs. Le second concerne l'action étatique avec une attention particulière portée à deux aspects : un aspect normatif et un aspect administratif-exécutif. L'habileté des entrepreneurs dans le champ des activités illicites a sa spécificité. La spécialisation dans les activités de recyclage, en rapport avec les transporteurs de drogue, en contact avec les opérateurs bancaires et financiers pour blanchir l'argent sale, donne lieu à l'acquisition d'un savoir-faire des entrepreneurs, entendu comme capacité d'identifier les secteurs économiques les plus profitables vers lesquels orienter les investissements. Mais il s'agit de capacités qui ne sont pas utiles pour créer des entreprises à fonction productive, sinon dans un but limité de connexion entre l'offre de biens illégaux et l'exploitation, à but économique, de liens financiers et politiques en vue d'obtenir l'accès aux financements publics et aux adjudications. Ainsi les capacités managériales se forment-elles essentiellement en relation à la politique, dont le rôle est excessivement "dramatisé". Les disponibilités d'argent liquide ne sont pas dirigées pour promouvoir le développement économique, mais s'orientent vers d'autres activités. Non seulement les capitaux ne s'accumulent pas dans les "mains adéquates"[A. Gerschenkron, Economic Backwardness in Historical Perspective, Cambridge (Mass.), Belknap Press-Harvard University Press, 1962.], mais les conditions sociales pour que se créent ces "mains adéquates" n'existent pas. Tant que les raisons de convenance économique à investir dans le champ des activités illicites persistent, il reviendra à ce secteur de se développer et à cette forme d'entreprise adéquate de se reproduire. L'effet de complémentarité des investissements fonctionnera en fait pour ce secteur[Sur cet effet, voir A. Hirschmann, The Strategy of Economic Development, New Haven, Yale University Press, 1958.]. Il est vrai qu'opérer dans le secteur de l'économie illégale comporte des coûts potentiellement très élevés ; et, en premier lieu, la possibilité d'être découverts et de voir disparaître en un instant la richesse accumulée. Mais si les risques sont réduits grâce à des stratégies de 
couverture et de dissimulation, alors la possibilité de profits immensément plus élevés que ceux qui peuvent être réalisés en investissant dans des activités licites est une incitation plus que suffisante pour orienter les individus vers le secteur illégal. La persistance de formes de protection/extorsion ou de violence, de la part de la criminalité organisée, concourt en outre à conditionner négativement la possibilité de voir émerger des capacités d'entreprise orientées vers des activités productives dans le secteur de l'économie licite. La persistance de la violence sur le marché décourage les investissements éventuels, réduit la gamme des entrepreneurs potentiels et pousse ceux qui veulent entrer dans l'arène économique à s'équiper des mêmes armes que les groupes mafieux. Donc en définitive, l'extension de la violence sur le marché fait obstacle au développement d'attitudes conformes aux lois normales du marché; elle favorise des orientations caractérisées par la logique du vol et par des manières de prédateurs. Ainsi, la prévisibilité générale du marché comme condition de la rentabilité de l'entreprise est fortement perturbée et ceci décourage d'entreprendre des activités économiques dans le secteur légal, soit en raison des profits plus bas par rapport au secteur illégal, soit en raison des coûts additionnels plus élevés (nécessité de payer la protection-extorsion), soit enfin en raison du risque de devoir fermer l'activité à cause de la violence socialement diffuse. La diffusion de la criminalité organisée provoque ce type de risques même pour les activités économiques normales, comme le montrent les tristes événements des régions méridionales d'Italie. Dans la dernière décennie, la croissance de l'emploi industriel s'est vérifiée seulement dans les régions où une forte présence de la criminalité organisée ne s'enregistre pas.

\section{LES POLITIQUES PUBLIQUES}

Si ce sont bien ces raisons liées à la présence de la criminalité organisée qui déterminent les tendances à la croissance des trafics illicites ou au découragement des initiatives légales d'entreprise, comment pèsent les politiques pour conditionner ou favoriser ces tendances ? Je cherche ici à démontrer brièvement que l'hyperlégislation, la sous-exécution, et plus encore, une combinaison des deux, ont une incidence sur le rapport entre croissance des trafics illicites et croissance de la régulation sociale par la violence. Mais voyons tout d'abord comment l'hyperlégislation peut influer sur l'extension de la sphère même des trafics illicites. Nous savons que c'est cette configuration juridico-normative des comportements de la part de l'Etat qui définit les catégories du licite et de l'illicite. Chaque fois que l'Etat intervient de façon normative pour établir qu'un comportement déterminé est juridiquement passible d'une sanction pénale (ou administrative), il étend ainsi la sphère des comportements (pénalement ou administrativement) illicites. Tout ce qui n'est pas objet d'intervention normative de l'Etat est indifférent à cette distinction et, en conséquence, "est juridiquement licite tout ce qui n'est pas expressément prohibé". L'extension progressive des tâches de l'Etat dans les sociétés contemporaines a comporté une tendance à l'extension de la sphère de l'intervention normative, et donc une extension de cette catégorie même de comportements illicites. D'autre part, il y a des comportements qui, avec le temps, perdent de leur importance sociale comme délits et sont dépénalisés. Il existe pourtant des cas dans lesquels l'extension juridique de la sphère de l'illicite se traduit par une série de véritables effets pervers. Il s'agit de tous ces phénomènes où la force des 
comportements sociaux est plus grande que la répression prévue par la loi, et pour cela, celle-ci en vient à revêtir la qualité de simple sanction morale (qui est contraire à la fonction laïque du droit pénal moderne). L'exemple classique a été la prohibition de l'alcool aux Etats-Unis. Précisément à propos de la prohibition, White[W. F. White, Street Corner Society , Chicago, University of Chicago Press, 1947.] expliquait comment elle constituait la base pour la formation des organisations de racket urbain à Connerville, exemples classiques du power syndicate. On peut dire la même chose aujourd'hui en Italie de la loi Jervolino-Vassalli sur la possibilité juridique d'infliger une peine aux toxico-dépendants, qui a eu et continue à avoir des effets pervers notables, notamment l'extension de la régulation par la violence. L'erreur principale commise par cette loi a été de retenir que l'on pouvait toucher le trafic de drogue en agissant sur la demande, sans au contraire comprendre qu'il s'agit substantiellement d'un marché de l'offre, dans lequel la demande est inélastique. Sur la base de ce présupposé, on a en substance assimilé au trafic la détention d'une dose supérieure à la moyenne quotidienne, en introduisant la possibilité juridique d'infliger une peine aux toxicodépendants à égalité avec le trafiquant, pouvant aller de 8 à 20 ans de réclusion. L'application d'une peine identique pour les trafiquants et les consommateurs a eu l'effet de créer les conditions pour une plus grande complicité entre les deux catégories d'individus, tandis qu'une politique efficace contre la drogue devrait tendre à dresser le consommateur contre le trafiquant. En outre, en introduisant le critère de la dose moyenne journalière, on pousse le consommateur à un rapport quotidien, beaucoup plus constant qu'il ne l'avait auparavant avec le trafiquant, en l'exposant beaucoup plus qu'avant à entrer dans le circuit du trafic. Enfin, la loi n'a pas tenu compte de certains paradoxes typiques de l'offre de drogue. En particulier, quand la quantité de drogue disponible sur le marché se réduit, à cause, par exemple, de la confiscation des doses, les trafiquants tendent à rétablir la quantité originaire en la coupant de façon sauvage. Ceci expose le consommateur à de graves risques pour sa santé, cela le pousse à utiliser plus de doses puisque chacune a une moindre efficacité, cela le contraint à chercher la dose en dehors de son cercle de référence habituel, donc sur des marchés dont il ne connaît pas les caractéristiques. Une des conséquences en est l'augmentation des morts par overdose. Un tel phénomène est unanimement interprété comme un indicateur du développement de la consommation et il a été utilisée, à son tour, pour réclamer des politiques plus répressives contre la consommation alors qu'au contraire, elle peut être un indicateur non pas d'une augmentation de la consommation mais plus simplement du mécanisme à peine illustré de réduction de l'offre. Un autre défaut de la loi est constitué par le caractère complexe des normes qui prévoient des vérifications administratives ou à mi-chemin entre l'administratif et le pénal qui doivent être prises par le préfet, le juge de paix, etc., à l'égard du toxico-dépendant trouvé en possession d'une quantité supérieure à la dose moyenne quotidienne. Il s'agit substantiellement de prescriptions inapplicables car elles entraînent une surcharge impossible à soutenir par les autorités chargées de l'application de la loi. Pourquoi s'arrêter sur cette loi ? Parce qu'il s'agit d'un exemple classique d'extension abusive de la sphère de l'illicite pénal. Les principes modernes de laïcité du droit pénal dans les civilisations occidentales prévoient que ne peut être considéré punissable celui qui ne cause pas de dommage à autrui. Ne peut être poursuivi pénalement celui qui ne cause un dommage qu'à luimême. Ceci est un principe qui découle de la séparation entre le droit et la morale, lequel justement constitue le fondement de la laïcité du droit pénal moderne. La récente loi italienne sur la drogue non seulement a violé ce principe ; mais d'un point 
de vue technique, elle a constitué en outre un cas d'hyperlégislation qualitative accompagnée d'une sous-exécution. Situation typique où le gonflement normatif de la sphère de l'illicite entraîne la prolifération des activités économiques illicites, donc l'augmentation de la demande de "gouvernement criminel" de la criminalité et, en conséquence, la multiplication des power syndicates avec la régulation sociale par la violence qui en découle[Tandis que je rédige la version définitive de cette contribution (9 novembre 1992), les organes de presse publient la nouvelle que le premier ministre italien, Giuliano Amato, a déclaré son intention d'abolir la possibilité juridique d'infliger une peine au toxico-dépendant, en maintenant les sanctions administratives. Il s'agit d'une prise de position qui reconnait l'inefficacité de la norme précédente. Selon les dernières informations de la presse, les personnes actuellement en prison qui devraient être libérées à la suite d'une mesure de ce genre seraient 12.000. Ce chiffre est à mettre en relation avec l'énorme diffusion du sida dans les prisons italiennes et le danger croissant de contamination qui y existe à l'heure actuelle.]. Un autre cas d'hyperlégislation, cette fois dans le champ administratif et de type quantitatif, est constitué par la norme relative aux adjudications dans les travaux publics. Le nombre élevé et la complexité des voies et procédures nécessaires pour prendre une décision entraînent une situation de pouvoir discrétionnaire irresponsable au profit des hommes politiques et des fonctionnaires aux mains desquels réside le pouvoir de décider. Un pouvoir discrétionnaire se présente de fait dans la concession des adjudications, mais sans aucune responsabilité car la procédure administrative est scandée par une série de passages rigidement établis dans lesquels le pouvoir discrétionnaire de l'acteur n'est pas formellement prévu. Le nombre élevé des étapes à travers lesquelles doit passer le texte permet aux fonctionnaires et aux hommes politiques d'opérer des pratiques dilatoires d'obstruction. Dans des situations de ce type, il est facile pour les power brokers qui, comme nous le savons, sont entraînés à la manipulation des hommes et au contrôle sur les milieux, d'intervenir à travers l'arme de la corruption. Le droit des adjudications est un des points les plus délicats d'intervention possible d'une régulation violente. Les exemples ne concernent plus seulement l'Italie méridionale où l'augmentation $\mathrm{du}$ nombre $\mathrm{d}$ 'homicides d'entrepreneurs et représentants de gouvernements locaux perpétrés par la criminalité organisée enregistrée dans les années 80 est un indicateur pesant. Même en Lombardie et dans d'autres régions de l'Italie septentrionale, de graves préoccupations existent devant l'extension de la violence sur le marché. On soulignera que le véritable problème n'est pas tant constitué par les activités économique illicites, qui doivent être contrecarrées par des actions efficaces, que par leur traduction en demande de pouvoir. L'abbé Bernard de Mandeville[La fable des abeilles.Ou Vices privés, bénéfices publics, 1714.] soutenait qu'une société ne peut pas se gouverner sans l'action des voleurs, des prostituées et en général du crime. Il est peut-être difficile d'accepter un cynisme aussi apparent mais un sain réalisme doit nous faire retenir que la sphère de l'illicite ne peut absolument pas être éliminée puisqu'une partie est liée à l'activité normative même de l'Etat. Une politique réaliste est donc celle de la réduction du dommage. Pour ce faire, il est nécessaire de prêter une grande attention aux politiques publiques et d'éviter que l'hyperlégislation et la sous-exécution n'aggravent les problèmes au lieu de contribuer à les résoudre. 


\section{RÉSUMÉS}

Les activités illicites en Italie semblent avoir atteint, dans la dernière décennie, un niveau "critique". L'Etat italien n'a manifestement plus le monopole de l'usage de la violence sur son propre territoire. Toutefois, le développement de la criminalité dite organisée, qui rentre directement en concurrence avec l'Etat, au titre notamment d'entreprise de protection garante des biens et de la sécurité physique, n'est "possible" que si ce dernier est incapable de tenir et d'assurer sa situation de détenteur de monopole de la violence. L'existence de cette criminalité est inhérente avec celle de l'Etat. Elle ne constitue pas un anti-Etat et ne peut "prospérer" que lorsqu'il y a un Etat, mais inefficace. La criminalité organisée n'est donc point liée à la disparition de l'Etat, mais "seulement" à sa faiblesse. Les réponses étatiques (les politiques publiques) semblent par ailleurs en total inadéquation avec les problèmes liés à la criminalité organisée. La combinaison entre l'hyperlégislation et la sous-exécution ont une incidence déterminante sur le rapport entre croissance des trafics illicites et croissance de la régulation sociale par la violence.

\section{INDEX}

Index géographique : Italie

Mots-clés : drogue, mafias et crime organisé, violence, contrebande et trafic

\section{AUTEUR}

\section{RAIMONDO CATANZARO}

Traduit de l'italien par Isabelle Sommier. 